\title{
Damage Detection of Beam Bridge Under a Moving Load Using Auto-encoder
}

\author{
Juntao $\mathrm{Wu}^{1}$, Zhenhua $\mathrm{Nie}^{1,2}$ \\ 1. Jinan University, Guangzhou, 510632, China \\ 2. The Key Laboratory of Disaster Forecast and Control in Engineering, Guangzhou, 510632, China
}

\begin{abstract}
A novel damage detection approach based on Auto-encoder neural network is proposed to identify damage in beam-like bridges subjected to a moving mass. In this approach, several sensors are used to measure structural vibration responses during a mass moving across the bridge. An auto-encoder (AE) neural network is designed to extract features from the measured responses. A fixed moving window is used to cut out the time-domain responses to generate inputs of the AE neural network. Moreover, some constraints are applied on the hidden layer to improve the performance of the AE network in training process. When the training is complete, the encoder was regarded as a feature extractor. And the damage index is defined as the cosine distance between two feature vectors obtained from adjacent data windows. By moving the window along the measured vibration data, we can calculate a damage index series and locate the damage position of the structure. To demonstrate the performance of the proposed method, numerical simulation is carried out. The results show that the proposed method can accurately locate both single and multiple damages using acceleration response. It infers the proposed method is promising for structural damage detection.
\end{abstract}

Key words: structural health monitoring; deep learning; auto-encoder; moving load; damage detection

\section{Introduction}

Bridges are important transportation infrastructure and play an important role in social and economic development. As more and more bridges enter the aging stage, their operational safety should be taken seriously. In the last decades, Structural health monitoring (SHM), which was already widely applied in the field of aerospace, also carried out extensive research in the field of civil engineering. SHM provides a practical way to assess structural performance during the service life of a structure. It usually refers to the procedure of measuring of structural dynamic response through sensors, then monitoring the possible structural damage and finally evaluating the operating status of the structure.

Structural damage detection (SDD) is an important part of SHM. In practice, a real bridge may be equipped with several sensors to measure its vibration response. However, it is almost impossible to identify the damage by monitoring the time-domain data directly. The key issue to structural damage detection is to translate the response into damage sensitive features which can reveal the status of the structure.

One kind of damage sensitive feature is modal parameters, such as natural frequency, mode shapes or their derivative. The changes in these parameters with respect to the parameters identified for the undamaged case are used to recognize

Copyright (C) 2021 by author(s) and Frontier Scientific Research Publishing Inc.

This work is licensed under the Creative Commons Attribution International License (CC BY 4.0).

http://creativecommons.org/licenses/by/4.0/ 
the structural damage. Cawley and Adams (1979) firstly conducted related researches to identify damage on a beam bridge through the change of natural frequency. Pandey et al. succeed in locating damage in 1991 according to the mode shape curvatures. Shi et al.(2000) proposed a damage localization method based on modal strain energy, another derivative of mode shape. However, in practice, the number of sensors is limited, and the accurate finite element (FE) model is difficult to establish. Furthermore, the data may be affected by environmental and operational conditions, leading to poor performance of this kind of method.

In contrast to the methods mentioned above, another kind of methodology, named "Data-driven", has attracted more attention in recent years. These methods extract damage-sensitive features via only concentrating on the anomaly of the data instead of requiring a model of the structure. Cavadas et al. proposed the moving principal component analysis (MPCA) method to detect and locate the damage using response under moving-load. Hao and Bao successfully detect the damage of the subsea pipeline system by modeling the dynamic response using the autoregressive moving average (ARMA) model. Hou et al. analyzed the characteristics of the measured response under wavelet transformation. The results show that the wavelet coefficients can identify the damage occurrence moment. However, researchers are difficult to find out a damage-sensitive index. Some damage index have good performance in a specific structure may fail to recognize the damage in other types of structure. The choice of the damage index is very dependent on the experience of researches.

In recent years, artificial intelligent technology provides a new research direction for structural damage detection. ANN is regarded as a classifier and the dynamic parameters of a structure are used as inputs. G lin et al. proposed a method to diagnose the construction damages by BP neural network based on frequency change parameters. Zhang used the regular vectors of changes in modal flexibility and conducted damage identification of a simply supported beam. Li et al. proposed a method using damage pattern changes in frequency response functions (FRFs) as the inputs of BP neural networks to identify damage. Although there are many successful cases, however, the selection of the features heavily relies on the researcher's experience which may have a great influence on the performance of the neural network. Besides, these methods require damage labels as the output of the ANN. It is almost impossible to achieve in real engineering.

To address these limitations, Auto-encoder (AE), one type of unsupervised model in the deep learning field, is used to automatically extract features from the original response without damage labels. Deep learning is a kind of methods extracting useful information from complex and massive data by multiple process layers to analyze clustering data automatically. AE is a generative model, composed of an encoder and a decoder. Deep neural networks are usually used to model the encoder and decoder. AE has been widely used in the anomaly detection field. Sakurada et al. proposed a method that utilized the auto-encoder to detect subtle anomalies of mechanical components. Wang et al. utilized an improved Auto-encoder which can predict and reconstruct the data at the same time to detect anomalies of an industrial control system. Dong-wook used RNN Auto-encoder to identify Internal information leakage behavior. Structural damage detection can be regarded as a type of anomaly detection, indicating the auto-encoder is also promising in the field of SDD.

\section{Methods}

This paper proposes a deep learning based damage detection method to locate the damage of a beam bridge subjected to a moving load. Auto-encoder neural is utilized in this method to catch the abnormality involved in the responses caused by the damage. The Auto-encoder neural network is trained as a feature-extractor and it can locate the damage position by monitoring the trend of the features. Ma utilized Variational Auto-encoder to locate damage of a beam-like bridge. And the damage index is defined as Euclidean distance between the feature obtained from the first window and the other 
moving window.

In this article, an auto-encoder with two inputs is designed to process acceleration response and to extract features from the raw data. The proposed method mainly consists of two steps: the training and the testing. The whole process of damage detection is shown in Figure 1. In the training step, the time-domain responses are sliced into little pieces by a fixed-length window. Two inputs of the auto-encoder are taken from two nearby data pieces. The training set is generated by data augmentation process, used for AE neural network training. Subsequently, the auto-encoder is trained on this dataset. When the training is completed, the encoder has the ability to extract features hidden behind the raw data. In the testing step, the response data is also sliced by a fixed-length window with chronological order. Then these pieces are inputted into the encoder so that the corresponding latent code can be obtained. Since the auto-encoder has two inputs at the same time, it also outputs two latent codes in each step. The distance between the latent codes get from nearby data pieces is considered as the damage indexes. By moving the windows along the time sequence, a time series of damage index is finally calculated. The damage index is supposed to have a sudden change at the position of the damage due to the discontinuity of the stiffness of the structure. Besides, it is worth noting that the proposed method just focuses on the change of the response, and the structural information is not necessary.

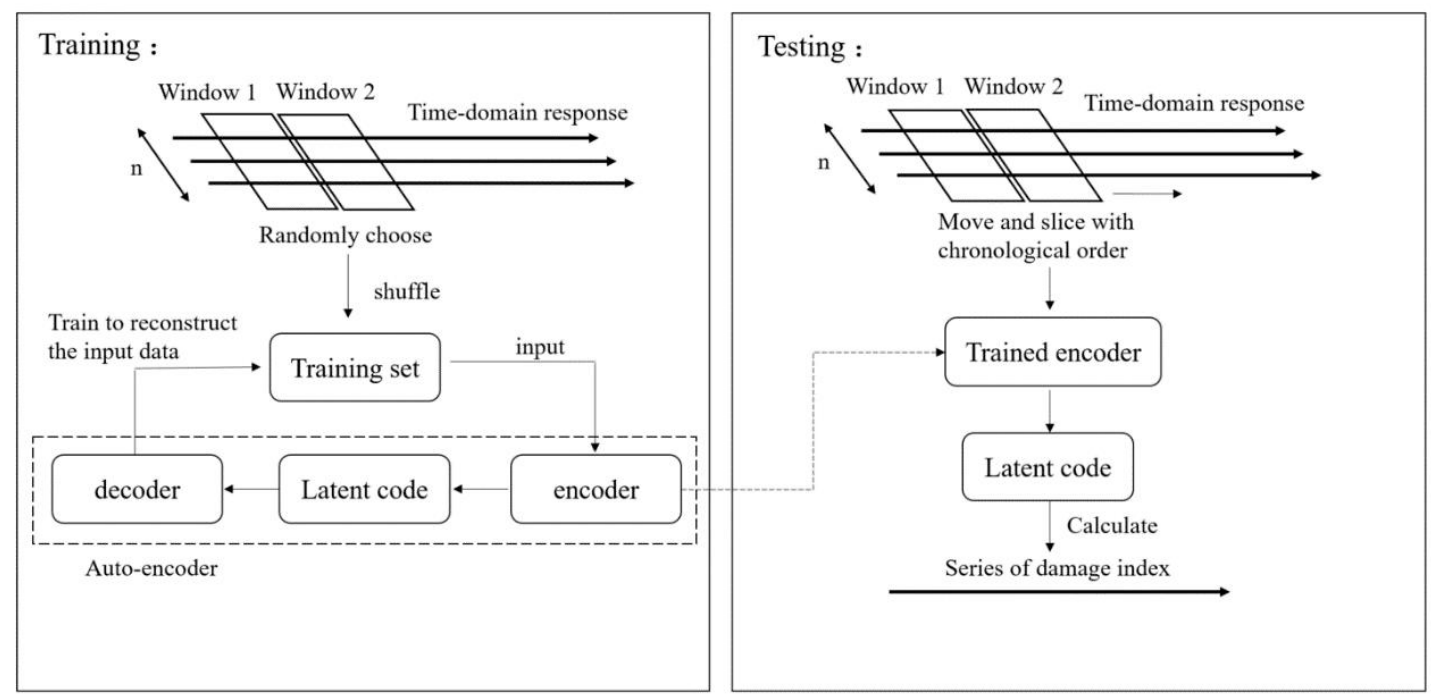

Figure 1. The overview of damage detection process

\subsection{Auto-encoder}

In this paper, auto-encoder is considered as a feature-extractor, which is trained by unsupervised learning to learn the reconstruction of its input data. An auto-encoder is composed of two parts: an encoder and a decoder, as shown in Figure 2. The encoder compresses the data and projects the data into a feature space. The decoder reconstructs the original data through the latent code extracted by the encoder. The latent code representing the features of the original data. An auto-encoder with a single hidden layer is shown as equation (1) and equation (2), respectively.

$$
\begin{aligned}
& Z=\sigma\left(W_{x z} X+b_{x z}\right) \\
& Y=\sigma\left(W_{z x} Z+b_{z x}\right) \\
& \text { err }=\|X-Y\|
\end{aligned}
$$

The encoder in equation (1) compresses the input $X$ to a hidden representation $Z$, called latent code, by a mapping following nonlinearity. The latent code is supposed carrying essential information of the original data. The decoder in equation (2) maps the latent code $Z$ back to the original input space as a reconstruction by the same 
transformation as the encoder does. The difference between the origin input $X$ and output $Y$ is defined as the reconstruction error as shown in equation (3). The auto-encoder learns the features of the original data by minimizing the reconstruction error.

By using the latent code of an auto-encoder as the input of another one, i.e., the auto-encoder will have multiple hidden layers, we can obtain a deep auto-encoder through stacking single-hidden-layer auto-encoders. A deep auto-encoder usually have better feature extraction capabilities. However, a deep auto-encoder is prone to overfitting, meaning it just mechanically copies the input to the output. Although the auto-encoder can reconstruct the training data very well, it has not learned useful data representation. Some constraints are usually applied to the hidden layer according to the specific problem to help the auto-encoder to achieve better performance. These constraints force the model to consider the relatively important parts of the input data, so that the essential features of the original data can be extracted.

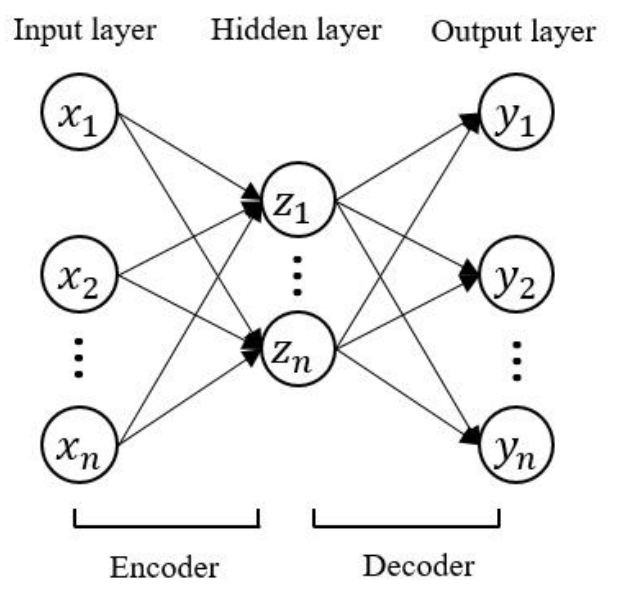

Figure 2. Auto-encoder with a single hidden layer

\subsection{Structural damage detection based on auto-encoder}

In this section, some training techniques used for $\mathrm{AE}$ training is introduced, including data pre-processing, data augmentation and $\mathrm{AE}$ neural network hyper-parameter settings.

\subsubsection{Data pre-processing}

For deep learning algorithms, normalization of the original data is benefit for speeding up the convergence of the model. In this paper, z-score normalization method is utilized to process the measured data, as shown in Eq.(4):

$$
\bar{x}=\frac{x-\mu}{\sigma}
$$

where $x$ is the original data set, $\bar{x}$ is the normalized data set, $\mu$ and $\sigma$ are the mean and standard deviation, respectively.

\subsubsection{Data augmentation}

For neural networks, the best way to improve model performance and model generalization is to expand the training set. However, in practice, the amount of available data is usually very limited. Data augmentation is an effective way to relieve the lack of data for deep learning algorithms. Data augmentation can generate more samples based on the true data. The data augmentation technique used in this paper are conducted as follows. In numerical simulation, each scenario has been simulated seven times. We randomly chose four measurements as the training set, two measurements as the verification set, and the remaining two measurements as the testing set. $n$ customized length data pieces are randomly sliced from the training set to build up a data batch, which is utilized to calculate the gradient of the loss function. The data augmentation process is conducted after data pre-processing. The whole process of data augmentation is shown in Figure 3. 


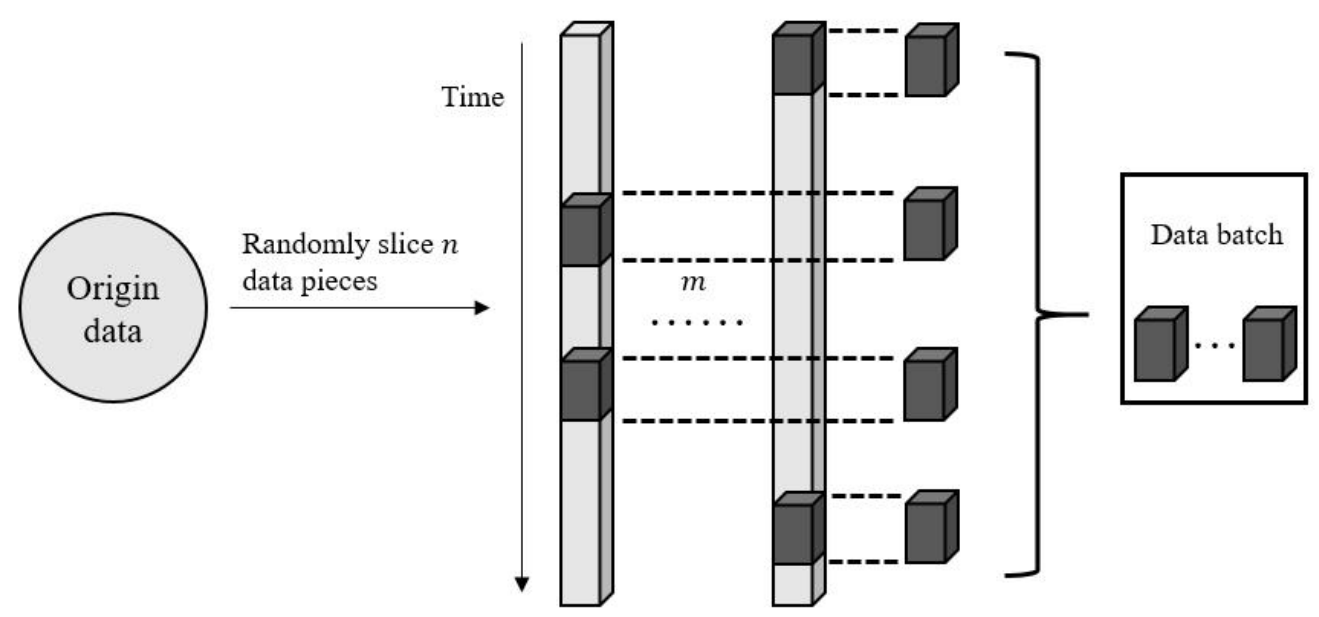

Figure 3. Data augmentation process

\subsubsection{The loss function}

As mentioned in section 2.1, a deep auto-encoder is prone to overfitting. The loss function of an auto-encoder neural network commonly only has a reconstruction term, which is usually mean squared error (MSE). MSE function, which refers to the expected value of the squared difference between two variables, is used to calculate the reconstruction error. A small value of the MSE function means a good fitting result of the neural network. Meanwhile, the latent code is presented as a vector, cosine function is used to calculate the similarity between the latent codes corresponding to different windows. The MSE loss function and the cosine function change during the training process as shown in Figure 4. After 200 epochs training, the MSE loss function converges to a stable minimum value, indicating the auto-encoder can reconstruct the original responses very well. The cosine function value declines at the beginning of the training, then increases to a high value and keeps until the training finished. Although the auto-encoder reconstruct the original responses well, but the cosine function value indicates that the latent codes corresponding to different windows are with a certain degree of difference. In other words, the auto-encoder just reconstructs the response well, but does not extract features with good performance. It is hoped that the latent codes keep a high degree of similarity while the bridge is undamaged. When the bridge was damaged, the damage can be identified through the sudden change of the latent code.

Based on the above discussion, there are two tasks for the auto-encoder: the first one is to reconstruct the original data and the second one is to make the latent code keep high degree of similarity. Thus the loss function is composed of two parts, the first part is MSE loss function. Since there are two inputs for the Auto-encoder, the MSE loss function is shown as Eq.(5):

$$
L_{M S E}=\left\|X_{1}-Y_{1}\right\|+\left\|X_{2}-Y_{2}\right\|
$$

where $X_{1}$ and $X_{2}$ are two inputs, $Y_{1}$ and $Y_{2}$ are reconstructed responses.

The second part of the loss function is the cosine function, which is used to evaluate the distance between the latent codes. This part of the loss function forces the auto-encoder to extract common features representing the damage condition and to ignore the difference caused by window selection. It is formulated as Eq.(6):

$$
L_{\text {COS }}=1-\cos \left(Z_{1}, Z_{2}\right)
$$

where $Z_{1}$ and $Z_{2}$ are latent codes corresponding to two windows. The total loss function is formulated as Eq.(7). $\lambda$ is a weight parameter to adjust the ratio of the two parts of the loss function.

$$
L=L_{M S E}+\lambda \cdot L_{C O S}=\left\|X_{1}-Y_{1}\right\|+\left\|X_{2}-Y_{2}\right\|+\lambda \cdot\left(1-\cos \left(Z_{1}, Z_{2}\right)\right)
$$



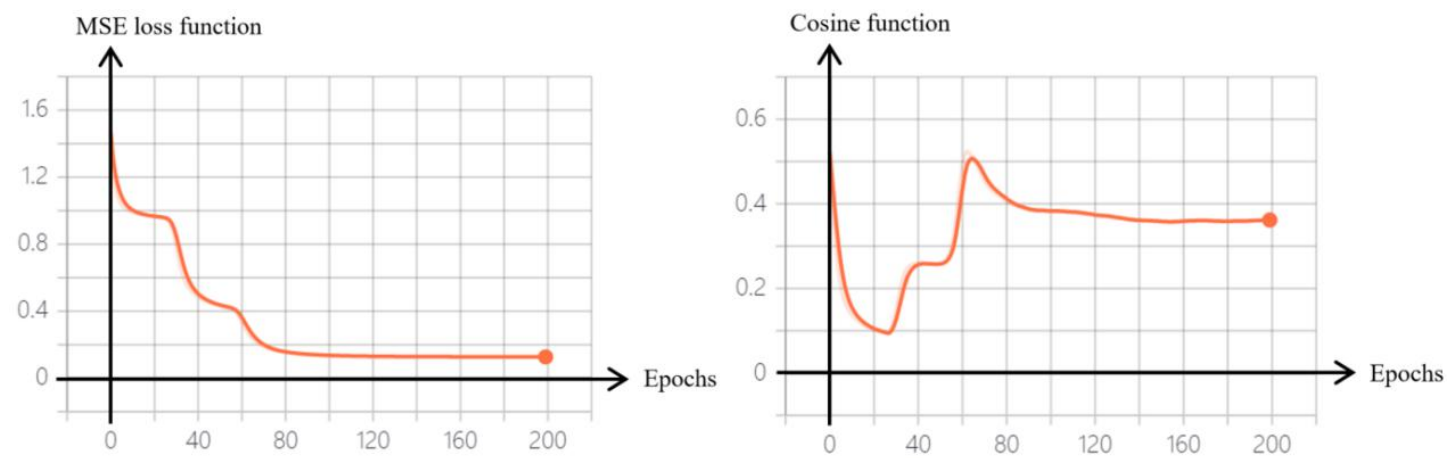

Figure 4. The loss function curve

\subsubsection{The length of the moving window}

A fixed moving window is utilized to slice the original data into pieces. On the one hand, the input dimension of the neural network should not be too large otherwise it will increase the computational cost. On the other hand, the data pieces should involve sufficient structural vibration information. According to Ma's suggestion the window length $l$ is defined as Eq.(8):

$$
l \geq 2 \frac{f_{s}}{f_{1}}
$$

where $f_{S}$ is the sampling frequency and $f_{1}$ is the fundamental frequency of the structure. Generally, the length of the input data usually set as a power-of-2, which may be convenient to set hyper-parameters of the neural network. Hence, the window length is determined to be larger than $2 f_{s} / f_{1}$ and the smallest value of power-of- 2 .

Fast Fourier transform (FFT) is used to calculate the fundamental frequency of the beam bridge. Therefore, the input of the Auto-encoder neural network is defined as:

$$
x_{l \times n}^{i}=\left[\begin{array}{cccc}
x_{i, 1} & x_{i, 2} & \ldots & x_{i, n} \\
x_{i+1,1} & x_{i+1,2} & \ldots & x_{i+1, n} \\
\ldots & \ldots & \ldots & \ldots \\
x_{i+l-1,1} & x_{i+l-1,2} & \ldots & x_{i+l-1, n}
\end{array}\right]
$$

where $n$ is the number of the sensors, $i$ represent the $i$ th window. The input dimensions of the neural network is $l \times n$.

\subsubsection{Damage index}

As mentioned in section 2.2.2, the second part of the loss function forces the latent code in the same scenario to keep a high similarity. In other words, if the structure is undamaged, the similarity of the latent codes keeps in a high level. Once the structure is damaged, the latent code would have a sudden change due to the discontinuity of the stiffness at local damage area. The damage can be detected by observing the change of the latent code. Based on this, the damage index is defined as Eq.(9):

$$
D I_{i}=\cos \left(Z_{i 1,} Z_{i 2}\right)
$$

where $D I_{i}$ is the $i$ th damage index, $Z_{i 1}$ and $Z_{i 2}$ is the $i$ th latent code corresponding to two nearby windows. Damage index $D I$ is the cosine distance between two latent codes.

\subsubsection{The structure of Auto-encoder neural network}

The encoder network of AE is mainly constituted by one-dimensional convolutional layers which is suitable for processing time-domain data, aiming to extract features from the original data. Firstly, the input layer is used to receive 
the original data. The input shape of the input layer is $l \times n$, where $l$ is the length of moving window, $n$ is the numbers of sensors. There are two windows inputting into the neural network at the same time. They share the same neural network and the calculations in the neural network are all performed at the same time, as shown in Figure 5. The encoder is modeled by Conv1D layer and Pooling layer. The latent code was outputted by the encoder. Then it pass through a flatten layer, therefore, we get a $1 \mathrm{D}$ vector from the encoder, representing the features extracted from the original data. Subsequently the decoder receives the feature vector and reconstructs the data. The decoding process is the reverse operation of the encoding process. Deconvolution layer and Upsampling layer are used instead of convolution layer and pooling layer. More details of the AE neural network will be illustrated in the next section.

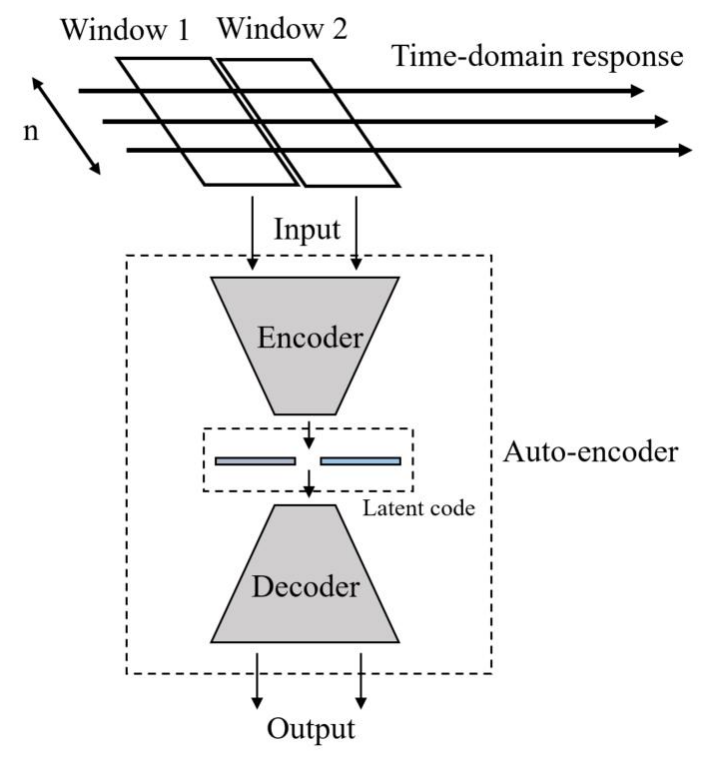

Figure 5. The structure of Auto-encoder neural network

\section{Numerical Simulation}

To demonstrate the effectiveness of the proposed structural damage detection method above, numerical studies are conducted in this section. Specifically, a simply-supported beam bridge subjected to a moving load is simulated using finite element analysis software ANSYS. Physical and material parameters of the beam model are shown in Table 1. The model is setting to be linear and elastic initial and after damaged. Seven sensors are uniformly placed on the top of the beam, diving the bridge into eight equal parts on average, as shown in Figure 6. A moving mass of 200kg passes through the bridge at a speed of $1 \mathrm{~m} / \mathrm{s}$ and the acceleration of seven sensors would be measured at the same time. The sampling frequency is $400 \mathrm{~Hz}$. Element No.4 is subdivided into 20 elements in the thickness direction. The simulation of different damage levels is conducted by deleting the sub-elements on the bottom edge of the beam. The damage is located at $L_{d}=0.4$. $\left(L_{d}=x_{d} / L\right.$ is the relative distance of the damage position from the left side of the beam). All damage scenarios are list in Table 2. In each scenario, five simulations are conducted. The severity of the damage is defined as the crack depth ratio in the direction of thickness of the beam. It should be noted that the response under the undamaged scenario is used for Auto-encoder neural network training. 


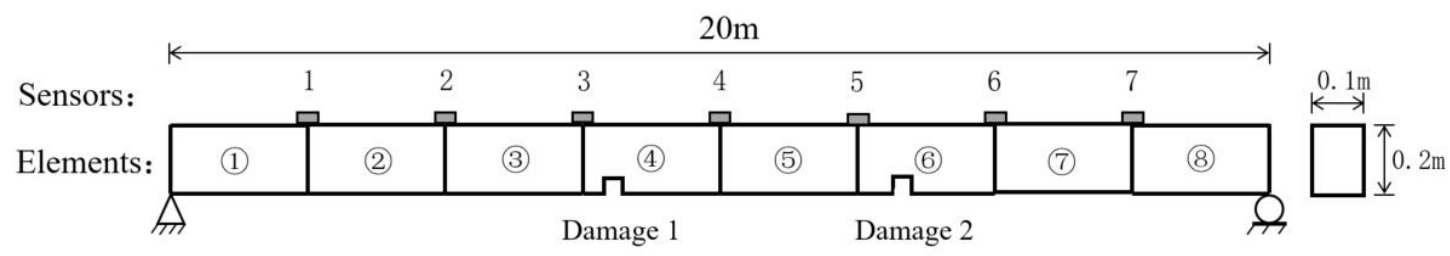

Figure 6. The beam model

Table 1. Physical and material parameters of the beam model

\begin{tabular}{cc}
\hline Parameter name & Value \\
\hline Beam length & $20 \mathrm{~m}$ \\
Section size & $0.2 \mathrm{~m} \times 0.1 \mathrm{~m}$ \\
Density & $7.85 \times 10^{3} \mathrm{~kg} / \mathrm{m}^{3}$ \\
Poisson ratio & 0.3 \\
Elastic modulus & $200 \mathrm{GPa}$ \\
Element type & Plane 42 \\
\hline
\end{tabular}

Table 2. Damage scenarios

\begin{tabular}{cccc}
\hline & Damage scenarios & Damage 1 (0.4L) & Damage 2 (0.7L) \\
\hline Single damage & 1 & - & - \\
& 2 & $10 \%$ & - \\
3 & $20 \%$ & - \\
& 4 & $30 \%$ & - \\
& 5 & $40 \%$ & $10 \%$ \\
& 6 & - & $20 \%$ \\
& 7 & - & $30 \%$ \\
Multiple damages & 8 & - & $40 \%$ \\
\cline { 2 - 4 } & 9 & $40 \%$ & $10 \%$ \\
& 10 & $40 \%$ & $20 \%$ \\
\hline
\end{tabular}

\subsection{Results}

When the moving mass passes through the bridge, 8000 data points are measured in 20 s by each sensor. Taking No. 2 sensor for example, the Fourier spectrum of the response was calculated, the fundamental frequency $f_{1}$ is $1.17 \mathrm{~Hz}$, as is shown in Figure 7. The length of the moving window can be calculated by Eq.(10):

$$
l \geq 2 \frac{f_{s}}{f_{1}}=2 \times \frac{400}{1.17} \approx 680 \quad(10)
$$




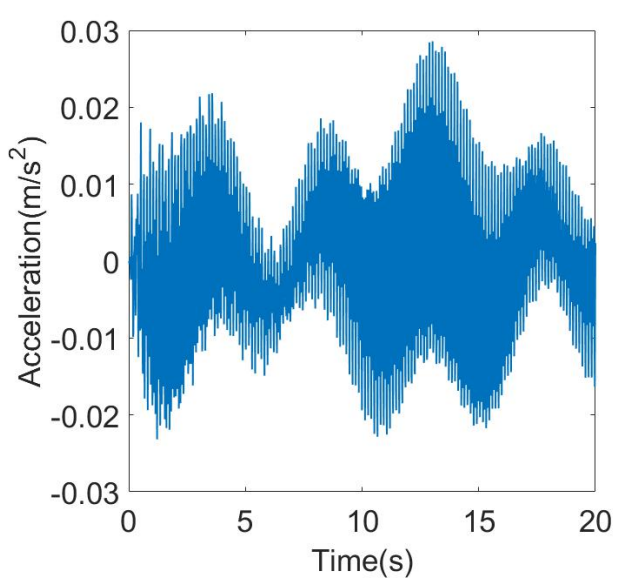

(a)

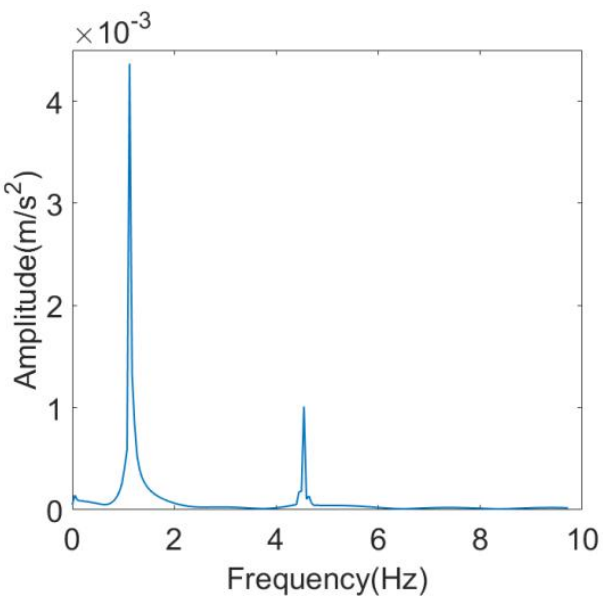

(b)

Figure 7. The response of sensor No.2: (a) Time history signal of sensor No.2; (b) The Fourier spectrum.

The length of the window should be the smallest value of power-of- 2 and larger than 680 . Thus, it is determined to be 1024. The moving window step is determined to be 32. The network layers settings of AE is shown in Table 3 . The ratio of the two parts of the loss function $\lambda$ is set as 0.1 . And SeLU activation function is used here. The RMSprop method is used as optimization algorithm. This algorithm can automatically reduce the learning rate during the neural network training to achieve better convergence results. The initial learning rate is set to 0.002 . After 200 epochs training, the loss function converge to a stable valve, as shown in Figure 8. That means the auto-encoder neural network is well-trained, and it can be applied to the testing step.

Table 3. Auto-encoder structure

\begin{tabular}{ccc}
\hline & Layer(type) & Output Shape \\
\hline Encoder & Input layer & $(1024,7)$ \\
& Conv1D_1 & $(1024,14)$ \\
& Maxpooling_1 & $(256,14)$ \\
& Conv1D_2 & $(256,21)$ \\
Maxpooling_2 & $(64,21)$ \\
Decoder & Conv1D_3 & $(64,28)$ \\
& Maxpooling_3 & $(32,28)$ \\
\cline { 2 - 3 } & Flatten_1 & $(896)$ \\
& Reshape & $(32,28)$ \\
& Upsampling_1 & $(64,28)$ \\
& Conv1D_6 & $(64,21)$ \\
& Upsampling_2 & $(256,21)$ \\
& Conv1D_7 & $(256,14)$ \\
& Upsampling_3 & $(1024,14)$ \\
& Conv1D_8 & $(1024,7)$ \\
\hline
\end{tabular}




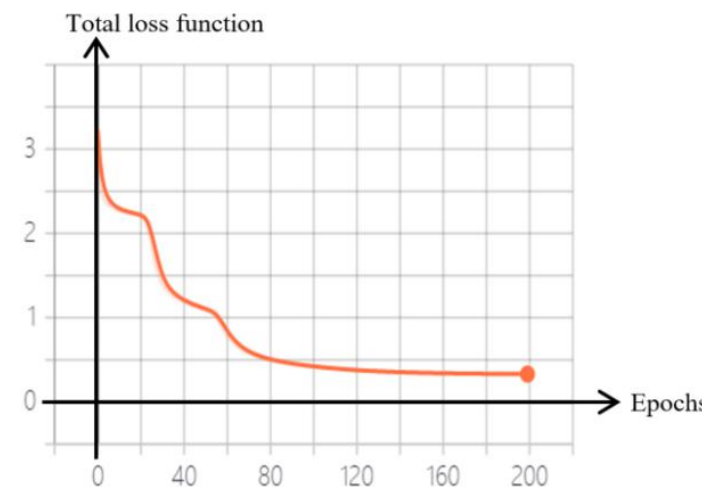

(a)

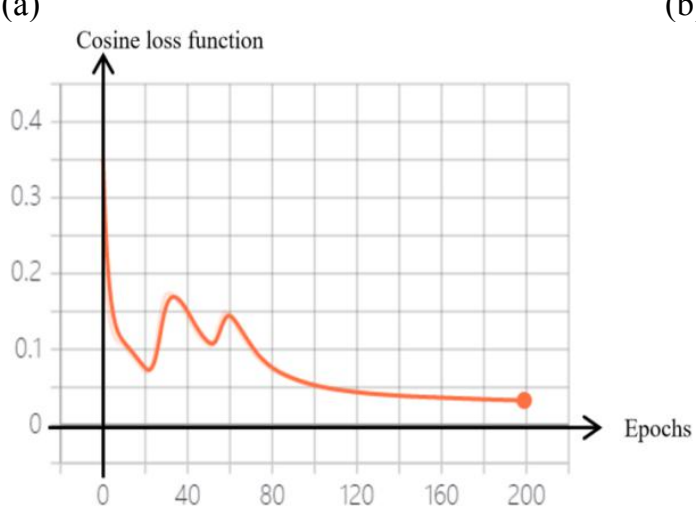

(c)

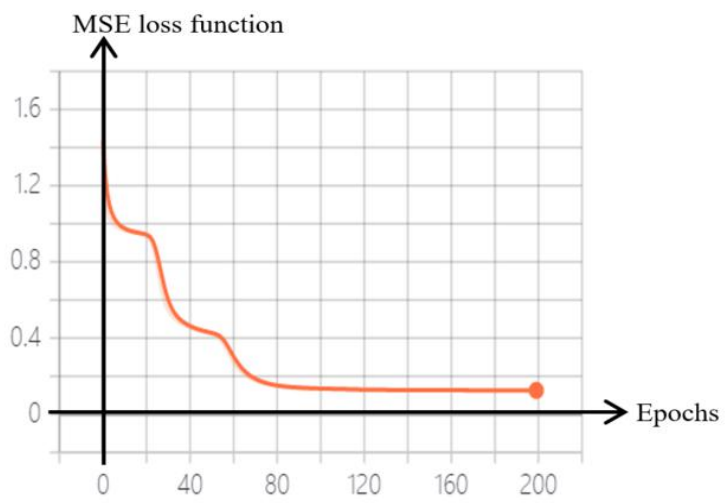

(b)

Figure 8. The loss function: (a) Total loss function; (b) MSE loss function; (c) Cosine loss function.

\subsubsection{Single damage}

The results are shown in Figure 9 and Figure 10. The responses under the undamaged scenario is used as training set. After the training, the encoder becomes a feature-extractor. Then it is applied in the testing step. In the scenarios with damage, the curve has an obvious peak at 0.4 and 0.7 , showing the excellent performance of the proposed method for the damage location. When the damage degree is low, as shown in Figure 7(a) and Figure 7(b), the sudden change on the curve is still obvious, and the damage can be located accurately. Besides, it is worth noting that the more serious the damage is, the lower the value of the damage index is. This can provide a reference for the estimation of the severity of damage. These results indicate that the proposed method can locate the damage accurately.

The auto-encoder neural network can well extract features with good performance of the responses. When bridge is damaged, the auto-encoder can immediately detect the abnormal vibration once the moving mass passes through the damage position.

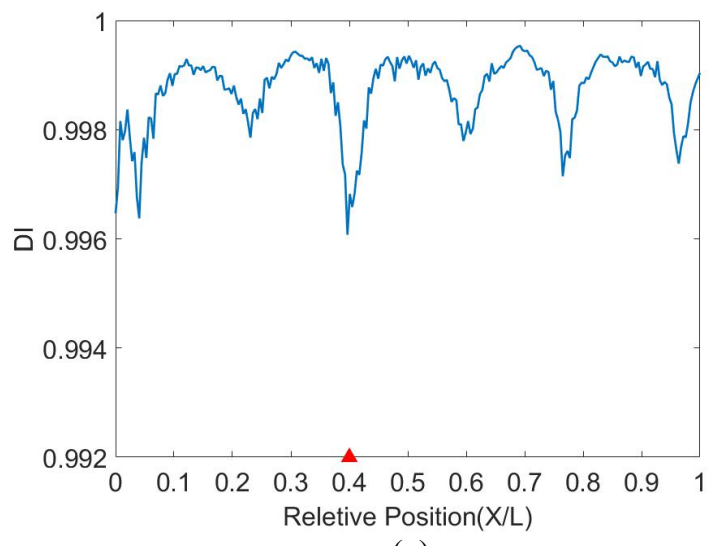

(a)

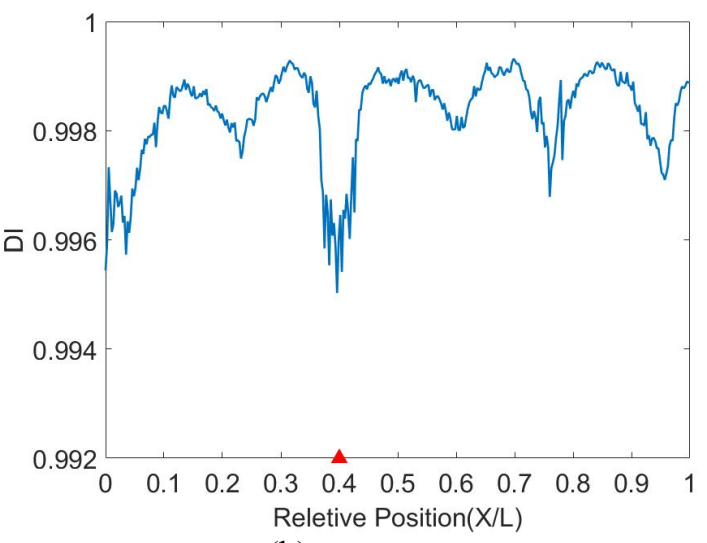

(b) 


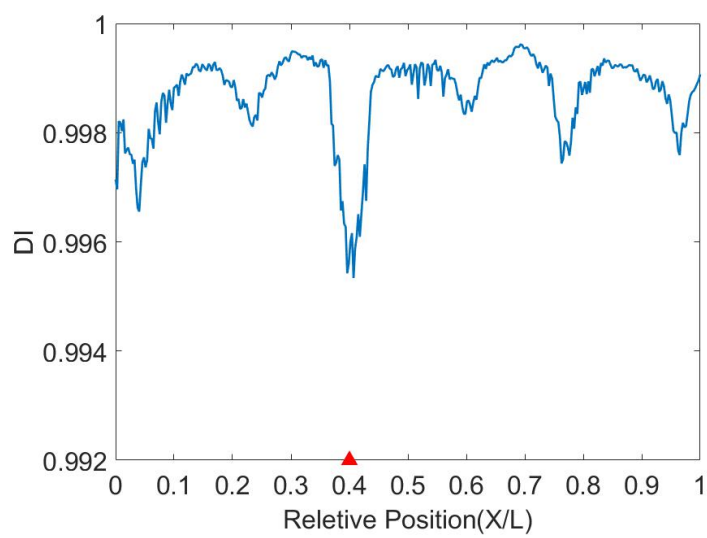

(c)

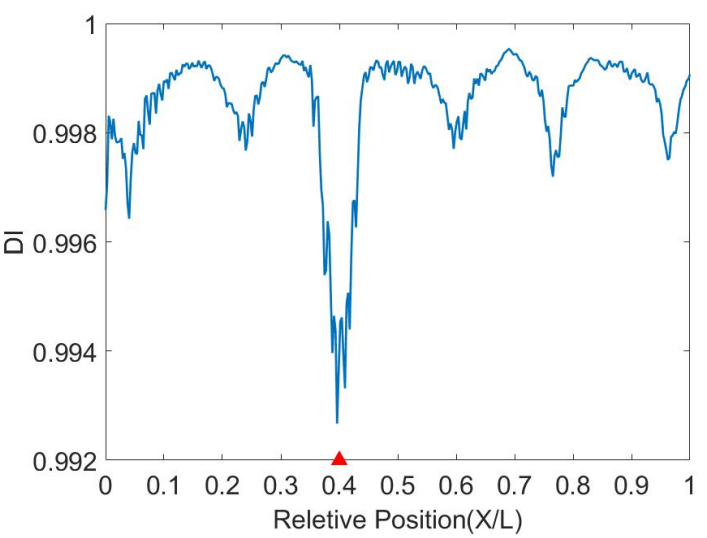

(d)

Figure 9. The results of single damage scenarios at $\mathrm{L}_{d}=0.4$ with damage severity: (a) $10 \%$; (b) $20 \%$; (c) $30 \%$; (d) $40 \%$.

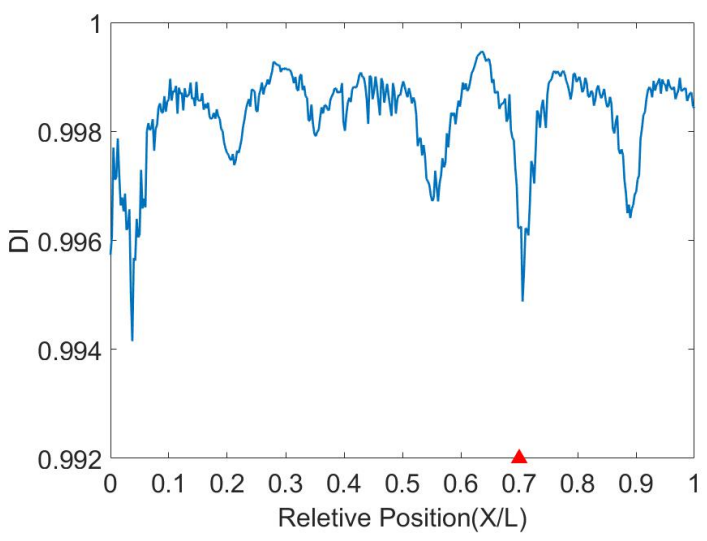

(a)

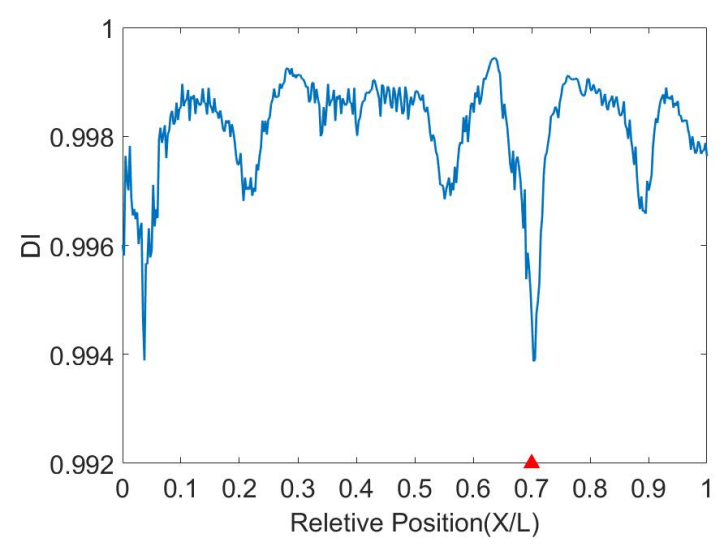

(c)

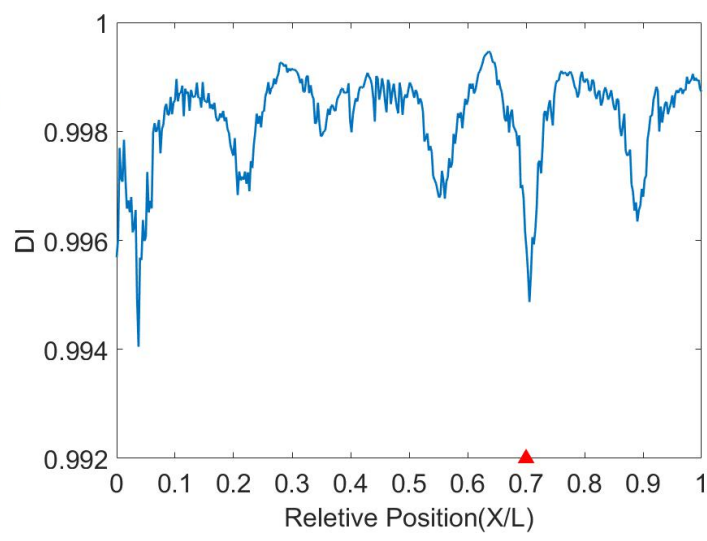

(b)

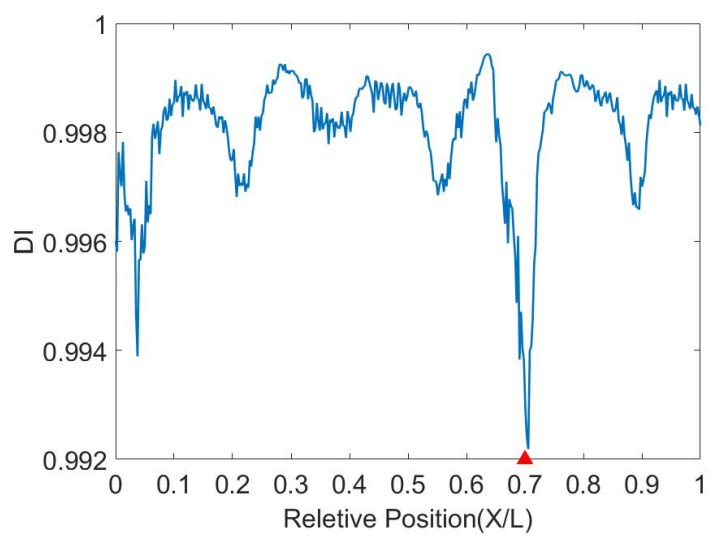

(d)

Figure 10. The results of single damage scenarios at $L_{d}=0.7$ with damage severity: (a) $10 \%$; (b) $20 \%$; (c) $30 \%$; (d) $40 \%$.

\subsubsection{Multiple damages}

Two damages were set on the bridge and the damage positions are $0.4 \mathrm{~L}$ and $0.7 \mathrm{~L}$, respectively. The damage severity in $0.4 \mathrm{~L}$ is set up to $40 \%$, and the damage severity in $0.7 \mathrm{~L}$ is set up to $10 \%$ and $20 \%$. In the previous section, single damage on the bridge has been identified confidently, the same auto-encoder model will be used to verify the effectiveness of multiple damages identification. The training and the testing step is repeated in these cases.

As shown in Figure 11, both damages at $0.4 \mathrm{~L}$ and $0.7 \mathrm{~L}$ can be identified. The trough at $0.4 \mathrm{~L}$ is very apparent as it is in single damage scenarios. The damage at $0.7 \mathrm{~L}$ also can be easily identified even though the damage severity is $10 \%$. The results show that the proposed method also has good performance in multi-damamges recognition. 

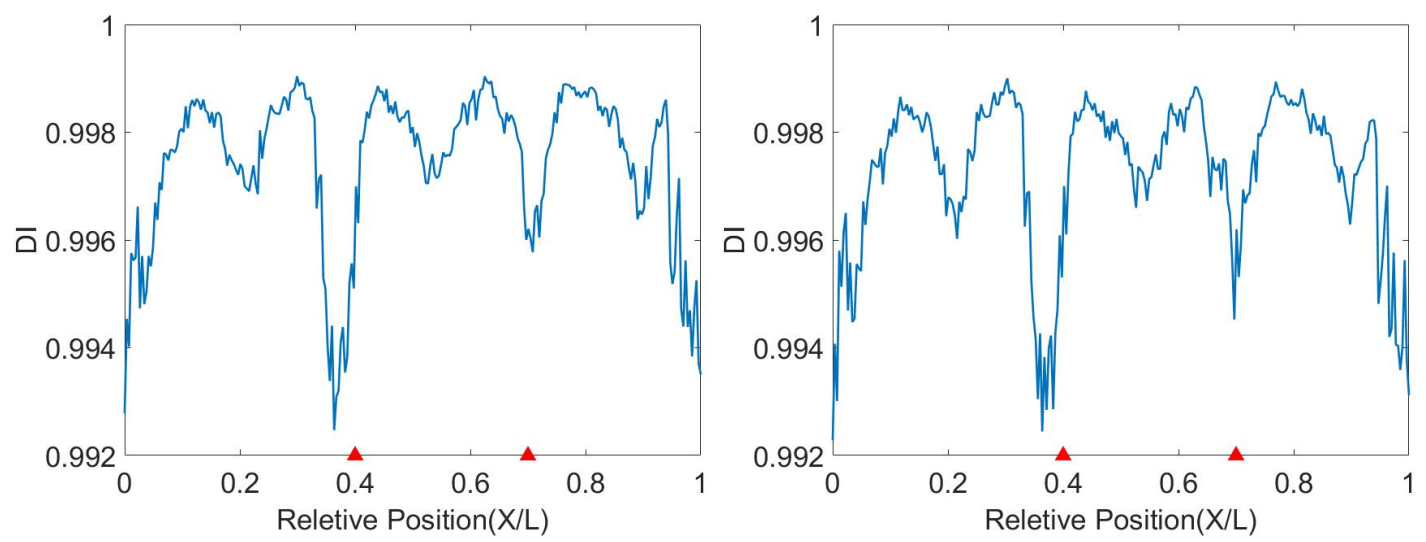

Figure 11. The results of multiple damages scenarios with damage severity: (a) damage 1: 40\%, damage 2: 10\%; (b) damage 1: $40 \%$, damage 2: $20 \%$;

\section{Conclusion and Discussion}

In this paper, a novel deep learning based damage detection approach using Auto-encoder neural network is proposed to detect the damage of beam bridge subjected to a moving mass. This method directly analyzes the measured acceleration responses of the bridge without the structure model information. Auto-encoder neural network is designed as a feature-extractor in this research, a fixed moving window is used to slice the responses to generate the inputs of the auto-encoder. Cosine distance loss function is applied in the training step to enhance the feature extracting ability of the neural network. A new damage index is proposed to locate the damage position of the bridge. Both single damage and multiple damages scenarios with different damage degrees are simulated. The results indicate that the auto-encoder can automatically extract damage-sensitive features from the original acceleration responses. It can accurately identify the damage location under both single damage scenarios and multiple damages scenarios. This method provide a new search direction in SDD. However, many factors have not been studied in detail, such as the speed, the weight of the moving mass and another factors in real engineering. Therefore, much more work should carry out to further verify the effectiveness of the proposed method.

\section{Conflicts of Interest}

The author declares no conflicts of interest regarding the publication of this paper.

\section{Acknowledgments}

The authors acknowledge the financial supports from the projects in key areas of Guangdong Province (No. 2019B111106001) and National Key Research and Development Project of China (No. 2019YFC1511000).

\section{References}

[1] Zaman D, A A li, Singh A. (2007). Structural Health Monitoring of Civil Infrastructure. International Journal of Advance Research in Science and Engineering, 5(3): 16-22.

[2] P. Cawley, R.D. Adams. (1979). The Location of Defects in Structures from Measurements of Natural Frequencies. Strain Anal. Eng. Des, 14 (2): 49-57.

[3] A.K Pandey, M. Biswas, M.M. Samman. (1991). Damage Detection from Changes in Curvature Mode Shapes. Sound Bib, 145(2): 321-332.

[4] Shi Z.Y., Law S.S., Zhang L.M. (2000). Structural Damage Localization from Modal Strain Energy Change. Journal of Sound and Vibration, 126(12): 825-844. 
[5] F. Cavadas, I.F.C. Smith, J. Figueiras. (2013). Damage Detection Using Data-driven Methods Applied to Moving-load Responses. Mechanical Systems and Signal Processing, 39(1): 409-425.

[6] Bao C.X., Hao H., Li Z.X. (2013). Integrated ARMA Model Method for Damage Detection of Subsea Pipeline System. Engineering Structures, (48): 176-192.

[7] Hou, Z.K., Noori, M. N. Amand, R. S. (2000). Wavelet-based Approach for Structural Damage Detection. Journal of Engineering Mechanics, 126(7): 677-83.

[8] Ye X.W., Jin T., Yun C.B. (2019). A Review on Deep Learning-based Structural Health Monitoring of Civil Infrastructures. Smart Structures and Systems, 24(5): 567-585.

[9] Lin G., Wei J. (2010). Structural Damage Detection Based on BP Neural Network Technique. IEEE.

[10] Zhang J. (2011). Structural Damage Detection Using Parameters Combined with Changes in Flexibility Based on BP Neural Networks. Advanced Materials Research, 243-249: 5475-5480.

[11] Li J., U. Dackermann, Xu Y.L., etc. (2011). Damage Identification in Civil Engineering Structures Utilizing PCA-compressed Residual Frequency Response Functions and Neural Network Ensembles. Structural Control Health Monitoring, 18(2): 207-226.

[12] Mayu Sakurada, Takehisa yairi. (2014). Anomaly Detection Using Autoencoders with Nonlinear Dimensionality Reduction. Proceedings of the MLSDA 2014 2nd Workshop on Machine Learning for Sensory Data Analysis, 4-11.

[13] Wang C., Wang B.L., Liu H.R., etc. (2020). Anomaly Detection for Industrial Control System Based on Autoencoder Neural Network. Wireless Communication and Mobile Computing, (02): 17-35.

[14] Dong-wook, Ha, K., Ryu. (2017). Detecting Insider Threat Based on Machine Learning : Anomaly Detection Using RNN Autoencoder. Journal of the Korea Institute of Information Security \& Cryptology, 27(4): 763-773.

[15] Ma X.R., Lin Y.Z., Nie Z.H. etc. (2020). Structural Damage Identification Based on Unsupervised Feature-extraction Via Variational Auto-encoder. Measurement, (160): 107811. 